\title{
Une école moderne, mais à la française
}

\section{Bernard Charlot}

\section{OpenEdition}

\section{Journals}

Édition électronique

URL : http://journals.openedition.org/ries/3172

DOI : $10.4000 /$ ries.3172

ISSN : 2261-4265

\section{Éditeur}

Centre international d'études pédagogiques

\section{Édition imprimée}

Date de publication : 1 septembre 1997

Pagination : 73-82

ISSN : 1254-4590

\section{Référence électronique}

Bernard Charlot, "Une école moderne, mais à la française », Revue internationale d'éducation de Sèvres [En ligne], 15 | 1997, mis en ligne le 04 juillet 2013, consulté le 19 avril 2019. URL : http:// journals.openedition.org/ries/3172 ; DOI : 10.4000/ries.3172

Ce document a été généré automatiquement le 19 avril 2019

(c) Tous droits réservés 


\title{
Une école moderne, mais à la française
}

\author{
Bernard Charlot
}

1 Tout au long des années soixante et soixante-dix, la France a débattu, sous forme médiatique, politique ou savante, de l'échec scolaire et des inégalités sociales face à l'école. Cette question était aussi en débat dans d'autres pays mais en France non seulement elle occupait une place prédominante mais en outre elle tendait à occulter tous les autres problèmes ; ce n'est pas un hasard si la France a été le terreau des sociologies de la « reproduction ».

2 Cette question n'a pas été résolue, même si des progrès ont été accomplis, et elle reste à l'horizon de nombreux débats actuels. Cependant, elle n'est plus traitée frontalement, comme elle l'était dans les années soixante et soixante-dix. L'opinion publique est aujourd'hui sensible à d'autres thèmes et les chercheurs ont construit d'autres objets. En outre, le débat sur l'éducation et sur l'école s'est diversifié. Quelles sont aujourd'hui les principales questions en débat en France ? Dressons-en d'abord l'inventaire.

3 L'objectif de « $80 \%$ d'une génération au niveau du baccalauréat », formulé en 1985 et au centre de nombreux débats à la fin des années quatre-vingt, ne soulève plus guère de discussions aujourd'hui. Toutefois, cette élévation générale du niveau de formation des jeunes Français pose plusieurs problèmes : celui de l'échec persistant d'une fraction de jeunes dès le collège (11-15 ans), celui de l'accès de nouveaux publics au lycée (15-18 ans), celui de la massification de l'université.

4 La question qui retient le plus l'attention des médias et qui entretient une inquiétude vive dans l'opinion publique est celle des violences scolaires, liée, sous diverses formes (y compris scientifiquement peu légitimes ou idéologiquement douteuses), à celle de l'école en banlieue, à celle du développement de dispositifs d'aide aux devoirs, à celle de l'intégration des jeunes issus de l'immigration et à un rebond du débat sur la laïcité.

5 Tout en étant sensibles aux questions précédentes, les autorités nationales et locales et les personnels enseignants débattent d'une question qui n'est guère prise en charge par l'opinion publique: celle de la territorialisation des politiques éducatives (déconcentration, décentralisation, zones d'éducation prioritaires...). 
6 Liée aux trois précédentes, la question de l'efficacité des politiques et des pratiques éducatives est l'objet d'un intérêt croissant et d'un investissement nouveau de la part des enseignants. La question de la formation des maîtres a été en discussion au début des années quatre-vingt-dix et elle reste latente mais aujourd'hui elle n'est plus vraiment au cœur des débats.

7 Une autre question mobilise les autorités et les chercheurs et revient de façon récurrente dans les médias: celle de l'apprentissage et plus généralement de la formation en alternance. La France a développé, bien plus que d'autres pays, un appareil scolaire de formation professionnelle. Or, la France connait également un chômage des jeunes plus important que celui que l'on constate dans la plupart des pays européens. Aussi s'interroge-t-elle sur la pertinence de ce mode de formation professionnelle -auquel certains opposent le modèle allemand de la formation duale.

8 Enfin, la France expérimente de nouveaux rythmes scolaires, qui font l'objet d'un débat latent dans la société française.

\section{Les spécificités françaises}

9 Les questions dont nous avons dressé l'inventaire se croisent, s'articulent, s'appellent les unes les autres. Ce ne sont pas, en effet, des questions purement techniques et pédagogiques mais des problèmes qui renvoient aux difficultés et aux contradictions que la société française et son école doivent aujourd'hui affronter. Certes, toutes ces questions sont également en débat, peu ou prou, dans d'autres pays européens. Mais dans chacun de ces pays elles prennent des formes particulières, liées aux spécificités sociales et culturelles que chaque pays a construites au cours de son histoire. Avant de reprendre chacune de ces questions pour la présenter de façon plus détaillée, il nous faut donc expliquer quelles sont les spécificités du contexte scolaire français.

Première spécificité: la question de l'éducation présente en France une dimension éminemment politique. Le pouvoir royal, les révolutionnaires de la fin du XVIII siècle, Bonaparte, la République : tous les pouvoirs que la France a connus ont traité l'éducation comme une affaire d'État. Une configuration sociopolitique forte, portée à la fois par la bourgeoisie progressiste et par le mouvement populaire et soutenue par une alliance étroite entre l'État et les enseignants, s'est mise en place à la fin du XIX ${ }^{e}$ siècle : elle lie l'éducation aux droits de l'Homme, à la Raison, au Savoir, à la Nation, à la Laïcité. Aujourd'hui encore, l'éducation est une question politiquement très sensible en France : à deux reprises, depuis 1984, la question des rapports entre école publique et école privée a mobilisé des centaines de milliers de manifestants dans les rues du pays (défendant dans un cas la « liberté » d'enseignement et dans l'autre l'école publique); le poste de ministre de l'Éducation nationale est d'ailleurs considéré en France comme un poste très exposé, susceptible de ruiner la carrière politique de celui qui l'occupe.

11 Deuxième spécificité du contexte scolaire français : depuis les années soixante, il existe un lien étroit entre le niveau de scolarisation atteint par un jeune et son niveau d'insertion sur le marché du travail. L'État gaulliste s'est très impliqué dans le développement économique du pays au cours des années soixante et soixante-dix et il a voulu que le système scolaire, très centralisé, contribue à ce développement économique ; cela s'est traduit, notamment, par le développement d'un enseignement technique et professionnel articulé sur l'enseignement général. Peu à peu, chaque profession, qu'elle 
soit agricole, industrielle, commerciale ou administrative, a été dotée de diplômes permettant d'accéder aux différents échelons de la profession : ainsi, il existe en France un certificat d'aptitude professionnelle de barman.... Mais pour autant, la société française, qui reste très hiérarchisée, continue à privilégier les fonctions de gestion, de direction, de conceptualisation et les diplômes d'enseignement général qui y conduisent l'orientation vers les formations techniques, et surtout professionnelles, s'opérant sur la base de l'échec scolaire. Ainsi, le système scolaire français, tout à la fois, est très élitiste et concurrentiel et constitue une voie de passage quasi obligé vers le marché du travail, si modestes que soient les activités professionnelles exercées par les jeunes. La dimension politique de l'éducation prend alors une forme nouvelle: ce ne sont plus les valeurs à transmettre aux enfants qui sont l'enjeu fondamental du débat mais l'égalité des chances, c'est-à-dire la possibilité pour chaque enfant, quelle que soit son appartenance sociale, de bénéficier d'une mobilité ascendante grâce à l'école.

C'est dans ce contexte que survient la « crise » des années soixante-dix. Elle va produire de multiples effets, qui permettent de comprendre pourquoi ce sont les questions dont nous avons dressé la liste qui sont aujourd'hui en débat.

\section{Objectif : $80 \%$ niveau bac}

13 A cette crise, la France, après quelques hésitations, répond par ce que l'on peut considérer comme le troisième grand bond en avant du système scolaire : la fin du XIX ${ }^{\mathrm{e}}$ siècle et le début du XXe ont vu le développement et la généralisation de l'école primaire; les années soixante et soixante-dix ont permis de scolariser tous les jeunes au collège, premier cycle de l'enseignement secondaire; l'objectif de la fin des années quatre-vingt et des années quatre-vingt-dix est de conduire $80 \%$ des jeunes au lycée, second cycle de l'enseignement secondaire, et une proportion importante d'entre eux à l'université. Cette ambition prend appui sur des arguments liés aux évolutions économiques et techniques de la société moderne : face à la compétition internationale et à la concurrence des pays à main d'œuvre bon marché, un pays comme la France doit investir dans la technologie et donc dans la formation. Mais cette réponse s'enracine plus profondément dans la tradition française: lorsqu'un problème national est posé, le pays se tourne vers l'éducation.

14 La fin des années quatre-vingt et le début des années quatre-vingt-dix ont été marqués par le débat sur les « $80 \%$ d'une génération au niveau du baccalauréat », slogan lancé en 1985 par J.-P. Chevènement, ministre de l'Éducation nationale. Un ensemble de mesures officielles et de pressions institutionnelles ont été mises en œuvre afin d'atteindre cet objectif : révision des procédures d'orientation, construction de lycées, création d'un baccalauréat professionnel, institution de formes nouvelles de suivi et de soutien scolaire. Cette politique volontariste a produit des effets : alors qu'environ $35 \%$ d'une génération atteignait le niveau bac en 1984, le taux est aujourd'hui d'environ $63 \%$. L'objectif des $80 \%$ ne sera pas atteint en l'an 2000 mais celui des $70 \%$ peut l'être: le système scolaire français a réussi ce bond en avant que beaucoup jugeaient impossible et du même coup le débat s'est apaisé. Mais il s'est également déplacé.

Premièrement, ce progrès a aussi produit des effets pervers. D'une part, le volontarisme institutionnel a parfois fait confondre niveau de formation et passage dans la classe suivante: certains élèves entrent dans des classes sans avoir le niveau requis pour y réussir. D'autre part, que deviennent les $20 \%$ qui n'ont pas accédé au niveau 
baccalauréat ? Il y a eu un débat assez vif sur ce point, certains reprochant aux autorités d'abandonner un cinquième de chaque génération. En réalité, un objectif a également été fixé pour ces $20 \%$ : ils doivent recevoir une formation professionnelle (de niveau fin de collège plus deux ans). Mais il n'en reste pas moins que la situation sur le marché du travail des jeunes les plus fragiles, fussent-ils dotés d'une formation professionnelle de base, devient plus difficile quand $80 \%$ de leur génération a atteint un niveau bac. Enfin, la réussite même de cette politique volontariste pose des problèmes : que faire de ces jeunes mieux formés et soucieux d'obtenir un emploi correspondant à leur formation, dans une société où les emplois deviennent plus rares et l'embauche des jeunes plus difficile?

Deuxièmement, des problèmes se posent également en amont et en aval du lycée. Pour conduire $80 \%$ des jeunes au niveau du baccalauréat, encore faut-il qu'ils entrent au lycée dotés d'un niveau correct. De sorte que cet objectif, qui concerne directement les lycées, pose aussi le problème de la réussite des jeunes en amont et notamment au collège. Or, une frange significative de jeunes est en échec au collège : 10\% des jeunes environ sortent de l'école sans formation générale suffisante et sans réelle formation professionnelle. Un autre problème se pose, en aval du lycée, pour ceux qui ont réussi cette fois : les jeunes dotés d'un baccalauréat veulent continuer des études. Peu nombreux sont ceux qui parviennent à entrer dans les Grandes Écoles, secteur élitiste de l'enseignement supérieur; un réel effort a été consenti pour développer les formations technologiques en deux ans au-delà du bac mais elles ne peuvent pas pour autant accueillir tous les candidats; aussi un flux de jeunes bacheliers se déverse-t-il à chaque rentrée dans les disciplines générales de l'université. La France compte aujourd'hui plus de deux millions d'étudiants, dont environ un million et demi dans les universités (en 1980-1981: 1,2 million, dont 0,8 dans les universités). En quinze ans, le nombre d'étudiants accueillis dans les universités a presque doublé. Cette «massification » de l'université pose de redoutables problèmes : la question du financement des universités et surtout celle de l'échec (massif) au cours des deux premières années sont aujourd'hui en débat; en outre, le taux de chômage des diplômés universitaires a beaucoup augmenté ces dernières années (tout en restant inférieur à celui des non diplômés ou des diplômés d'un niveau moindre) et il y a là un sujet de grande inquiétude pour les classes moyennes. La question est posée notamment d'une professionnalisation plus grande des études universitaires.

\section{Crise de l'emploi, crise de légitimité de l'école}

17 Une autre grande question articulant le problème de la formation et celui de l'emploi agite la société française depuis quelques années : comment doit-on assurer la formation professionnelle des jeunes? Est-ce dans des établissements scolaires, comme c'est massivement le cas actuellement, ou par la voie de l'apprentissage (relativement peu développé en France, où il y a moins de 300000 apprentis) ou encore par un système d'alternance?

La France connaît un chômage des «jeunes » (d'abord catégorisés comme "16-18 ans » puis comme "16-25 ans ») beaucoup plus important que dans d'autres pays européens. Pourquoi? La question est très controversée et est l'objet d'âpres débats. La responsabilité du chômage des jeunes a souvent été imputée à l'école, selon un raisonnement implicite considérant que puisque c'est la formation qui mène à l'emploi, c'est l'insuffisance de formation qui mène au chômage. Cet argument n'est plus guère 
crédible car le chômage touche également aujourd'hui des jeunes dotés de diplômes professionnels et de diplômes universitaires de haut niveau. Dès lors, le débat s'est centré sur la question du mode de formation professionnelle: on a incriminé la formation professionnelle scolaire qui tient les jeunes trop éloignés des « réalités» du monde du travail et qui constitue un handicap pour accéder à l'emploi. A cette formation professionnelle en école, on oppose soit l'apprentissage soit une formation avec alternance de moments de type scolaire et de moments d'activité professionnelle.

Au-delà de ce débat sur les modalités les plus efficaces de la formation professionnelle et de l'accès à l'emploi, l'école française se trouve déstabilisée en profondeur par la crise de l'emploi. Les années soixante et soixante-dix ont peu à peu imposé une représentation de l'école en termes de positions sociales et d'insertion: l'école était importante pour le développement économique et social du pays, et, au niveau individuel, pour " avoir un bon métier plus tard ». Autrement dit, c'est la possibilité d'une mobilité professionnelle et sociale ascendante - et à tout le moins la certitude de trouver un emploi - qui conférait sa légitimité à l'école. Lorsque le pays affronte une crise de l'emploi, lorsque réussir à l'école ne garantit plus « un bon métier », ni même un emploi quel qu'il soit, l'école subit ellemême une crise de légitimité. Cette crise est d'autant plus forte que la société française doit affronter une contradiction qui devient de plus en plus vive : la France a répondu à la crise en allongeant la durée d'études d'une fraction de plus en plus importante de chaque génération, mais elle est de moins en moins en état de procurer à ces jeunes mieux formés un emploi stable correspondant à la formation reçue. Les jeunes doivent consentir de plus en plus d'efforts et de sacrifices pour réussir dans un système scolaire de plus en plus concurrentiel mais la récompense de ces efforts est de moins en moins désirable et de plus en plus aléatoire. Cette contradiction apparaît dans le discours même des jeunes, qui oscille entre "pourquoi travailler à l'école puisque même avec le bac on n'a plus rien? » et "donc il me faut un diplôme à un niveau bac plus deux». Les statistiques montrent une «course en avant» vers des diplômes de plus en plus élevés; mais cette course s'accompagne d'un sentiment de morosité et d'angoisse face à l'avenir.

\section{Des pratiques plus efficaces, un système moins centralisé}

La France doit par ailleurs affronter un autre problème, qui bouscule les traditions du service public d'éducation : celui de l'efficacité du système scolaire et de son pilotage. Des recherches récentes montrent que l'école française, contrairement aux représentations que l'on en a en France ou à l'étranger, a été historiquement portée par des dynamiques locales autant que par un volontarisme étatique. Mais il n'en reste pas moins que l'État français a toujours légiféré en matière scolaire, qu'il a incité, généralisé, contrôlé, qu'il s'est institué comme porteur de la parole légitime sur l'école. Du même coup, les enseignants, quelles que soient leurs pratiques réelles, et les différences entre ces pratiques, étaient censés mettre localement en application des programmes nationaux, selon des normes nationales, avec un contrôle national, pour former des enfants considérés comme semblables. C'est cette représentation et cette forme de légitimation de l'école qui ont commencé à se défaire en France.

21 Que ce soit dans les entreprises ou dans le secteur public, on demande aujourd'hui à ceux qui agissent non plus d'appliquer à des cas particuliers des règles générales édictées au 
sommet mais de régler des problèmes, tels qu'ils se posent localement. La légitimité n'est plus pensée en référence à la norme mais en termes d'efficacité. Une telle évolution frappe de plein fouet un système comme le système scolaire français, à la fois piloté du centre et pensé en termes de légitimité nationale. Elle engendre deux problèmes, qui font l'objet de débats.

Premièrement, on s'interroge désormais non plus sur la conformité des pratiques enseignantes aux textes mais sur l'efficacité de ces pratiques. Les établissements scolaires doivent établir des projets d'établissement prenant en compte les spécificités de leur public et de leur environnement ; les enseignants sont invités à pratiquer une pédagogie différenciée; une attention particulière et insistante est portée aux questions d'évaluation et une évaluation nationale des élèves est mise en œuvre au CE2 (troisième année de primaire), en $6^{\mathrm{e}}$ (première année de collège) et en $2^{\mathrm{de}}$ (première année de lycée). Les chercheurs sont invités, de façon parfois pressante, à étudier l'efficacité des pratiques, des enseignants, des établissements et, si possible, à identifier les facteurs d'une plus grande efficacité.

Deuxièmement, un système scolaire qui doit résoudre les problèmes là où ils se posent et dans les termes où ils se posent ne peut plus être intégralement piloté depuis le ministère de l'Éducation nationale. Il faut « territorialiser » les politiques éducatives, c'est-à-dire les définir en référence aux caractéristiques spécifiques des régions, des zones, des établissements eux-mêmes. Dès 1981 ont été créées des zones d'éducation prioritaires, où doit être mis en œuvre un principe de «discrimination positive " : les zones socialement défavorisées reçoivent des moyens supplémentaires et doivent élaborer un projet de zone; il existe aujourd'hui plus de 350 zones d'éducation prioritaires en France. A cette politique de zonage s'ajoutent les effets de la décentralisation des pouvoirs vers les collectivités territoriales : l'école était déjà en charge des communes, les collèges ont été confiés aux départements et les lycées aux régions. L'État, toutefois, conserve les instruments fondamentaux de régulation du système: définition des programmes, collation des diplômes, formation et rétribution des enseignants qui sont fonctionnaires d'État. Enfin, le niveau local est aussi celui de l'établissement qui, nous l'avons dit, doit concevoir un projet. À travers les zones, la décentralisation et les projets d'établissement, beaucoup, notamment dans le monde enseignant, voient se profiler le risque d'une segmentation et peut-être d'un éclatement du territoire scolaire national. La " désectorisation ", c'est-à-dire la possibilité de choisir pour son enfant un établissement secondaire autre que celui du secteur scolaire correspondant au lieu d'habitation, et la publication par les médias de palmarès des lycées accroissent les inquiétudes face à ce que certains perçoivent comme une dérive libérale et comme l'ébauche d'une école à plusieurs vitesses. Faut-il aller au-delà des mesures actuelles et territorialiser également les pratiques éducatives, les programmes, le recrutement et la formation des enseignants, voire leur traitement? La question est désormais posée en France, quoique d'une façon encore discrète.

Les enseignants français résistent à cette logique de l'efficacité (ou tout au moins au flou qui entoure cette notion) et plus encore à la segmentation sociale du système scolaire. Historiquement, ils ont construit leur identité en référence à l'Universel et à l'État-nation et on leur demande aujourd'hui de gérer l'hétérogénéité des élèves et de se penser comme des professionnels efficaces : c'est une véritable conversion identitaire qu'on tend ainsi à leur imposer. Ces enseignants, il est vrai, ressemblent de moins en moins à ceux que l'on a autrefois appelés les « hussards noirs de la République ». Ils sont aujourd'hui 
tous recrutés au niveau licence au moins $(\mathrm{bac}+3)$, y compris pour enseigner dans le primaire, où on ne les nomme plus « instituteurs » mais "professeurs d'école ». Ils sont formés dans des instituts universitaires de formation des maitres, créés au début des années quatre-vingt-dix. Après avoir connu quelques difficultés de mise en place et de vifs débats entre les tenants du Savoir et ceux pour lesquels l'enseignement est aussi un métier requérant une formation véritablement professionnelle, ces IUFM se sont stabilisés; pour autant, on ne peut pas considérer que la question de la formation des maîtres soit définitivement résolue et la tension demeure entre ceux qui valorisent la formation académique et ceux qui s'indignent d'une insuffisance de formation vraiment professionnelle.

\section{L'école en banlieue, la violence scolaire, le foulard islamique}

Plusieurs des questions que nous avons abordées convergent dans ce qui est aujourd'hui en France la question qui retient le plus l'attention des médias : la situation de l'école dans les quartiers dits "en difficulté » ou "sensibles». Il s'agit de quartiers souvent situés dans les banlieues des grandes villes, parfois dans les centres-villes dégradés de cités anciennes. L'inégalité sociale, en France comme ailleurs, tend à se spatialiser : les plus pauvres sont relégués, exilés, dans des zones de pauvreté où la qualité de l'habitat est médiocre, où les services publics et les transports sont insuffisants, où les taux de chômage sont plus élevés que la moyenne, où la proportion d'étrangers ou de nationaux issus de l'immigration est plus importante (mais il s'agit de quartiers inter-ethniques et non de ghettos au sens propre du terme). Ces zones sont aussi, comme on peut le deviner, des lieux où l'échec scolaire est plus fréquent et plus massif que dans d'autres quartiers et où se posent, de façon de plus en plus inquiétante, des problèmes de violences scolaires.

S'est ainsi constitué, au fil des années quatre-vingt, et accentué dans les années quatrevingt-dix, le problème de "l'école en banlieue ", qui est au cœur des débats médiatiques sur le système scolaire français. Plusieurs questions s'y croisent, et parfois s'y imbriquent. Jusqu'où faut-il adopter des politiques et des pratiques spécifiques dans ces zones? On ne peut certes y gérer l'école et y enseigner comme on le fait ailleurs mais une adaptation trop stricte à l'environnement et aux publics scolaires risque de fermer ces zones sur elles-mêmes et d'accentuer la segmentation du territoire scolaire national. Jusqu'où fautil accepter le partenariat de l'école avec des associations, des collectivités territoriales, des intervenants extérieurs et jusqu'où faut-il collaborer avec des dispositifs (associatifs ou municipaux) d'accompagnement scolaire et d'aide aux devoirs, qui prolifèrent actuellement autour de l'école? Certes, toutes les aides sont les bienvenues pour affronter des situations difficiles mais ne risquent-elles pas de déscolariser l'école, de lui faire oublier ses missions propres, de la délégitimer?

Par ailleurs (et c'est la question aujourd'hui centrale dans les médias et dans l'opinion publique), comment affronter, juguler, réduire la «violence " qui se développe dans l'école et autour de l'école? Certes, la France n'est pas comparable aux USA, les incidents graves restent rares et les meurtres exceptionnels. Mais ces incidents sont plus fréquents qu'autrefois, tout comme les intrusions dans l'établissement d'éléments violents externes à l'établissement (parents ou grands frères venus " régler des comptes » ou membres de bandes des cités environnantes); en outre, la violence est le fait d'élèves de plus en plus 
jeunes (les 8-12 ans suscitent des inquiétudes et les enseignants de maternelle eux-mêmes signalent une montée de l'agressivité chez leurs élèves). À côté de la violence proprement dite et des transgressions des règles de l'établissement se développent, non seulement dans les banlieues mais dans un nombre de plus en plus important d'établissements, l'absentéisme et les « incivilités » (les brimades et grossièretés diverses, ce que les Anglais appellent bullying ou mobing). Il y a là, peut-être, le phénomène le plus inquiétant à long terme: les règles de base qui constituent l'école en lieu spécifique et l'apprentissageenseignement en activité spécifique (venir à l'école, y travailler ou au moins y faire semblant de travailler, respecter les enseignants et les autres élèves, etc.) ont perdu de leur évidence ; on commence même à voir apparaitre en certains lieux une revendication des élèves d'être payés... Face à cette montée de la violence, des transgressions et des incivilités, l'école hésite: doit-elle "s'ouvrir sur son milieu» ou au contraire s'en protéger, y compris physiquement, et affirmer la spécificité et l'extra-territorialité du lieu scolaire?

Enfin, autour de la question de «l'école en banlieue» rebondit, sous une forme renouvelée, le problème de la laïcité. Il ne s'agit plus, comme autrefois, d'un conflit entre l'école privée et l'école publique ; ce conflit n'est pas mort mais il apparait aujourd'hui comme une sorte de conflit commercial entre deux réseaux scolaires, l'un majoritaire et l'autre minoritaire, argumentant en termes de concurrence déloyale de l'autre réseau. Le problème de la laïcité est désormais posé au sein même de l'école publique, à travers ce symbole (d'appartenance pour les uns et d'agression pour les autres) que constitue le «foulard islamique » dont se couvrent quelques jeunes filles (en nombre très réduit) de religion musulmane. Ce foulard a fait couler beaucoup d'encre, a produit des manifestations aux portes de certaines écoles, ou, à l'inverse, dans les salles de professeurs, a été l'objet de plusieurs décisions de justice (d'ailleurs contradictoires). La position officielle est textuellement très claire et pratiquement tout à fait ambiguë : le foulard est admis comme pièce vestimentaire et proscrit dès lors qu'il constitue un «signe ostentatoire » d'appartenance entrainant un prosélytisme religieux. Ce qui est ainsi en débat, sur le fond, c'est le strict maintien de la laïcité (définie comme absence de légitimité dans l'espace public de quelque différence que ce soit) ou au contraire l'acceptation souple d'affirmations communautaires au sein de l'école. Au-delà, l'enjeu est la capacité de l'école et des enseignants français à réaffirmer leurs ambitions universalistes tout en prenant en compte l'hétérogénéité accrue des publics scolaires. Il s'agit là d'un problème de fond, qui ne se limite pas à la question de la laïcité : le racisme progresse actuellement dans le corps enseignant français; certes, il reste le fait d'une minorité mais il a commencé à contaminer le corps professionnel qui, a priori, semblait le mieux immunisé contre cette forme de dégénérescence morale et sociale.

Tous les débats que nous avons évoqués tendent à interférer et tous convergent dans la question de « l'école en banlieue » qui produit une sorte d'effet-loupe.

Reste cependant une question régulièrement soulevée dans les médias, qui est l'objet d'expérimentations officielles, qui suscite un certain intérêt de l'opinion (sans passion toutefois) et qui pourtant ne nous semble pas étroitement liée aux précédentes : celle des rythmes scolaires, et tout particulièrement de l'organisation de la semaine et de la journée d'école. Si nous devions relier cette question aux précédentes, ce serait sur le mode de l'opposition, de la réaction : la pression scolaire exercée sur les jeunes par les parents et l'école, dans un contexte de concurrence de plus en plus sévère, oblige parfois à se rappeler, en réaction, que les élèves sont des enfants et des adolescents et qu'il n'est 
ni pertinent ni légitime que tout leur temps soit dévoré par la compétition scolaire. Le débat sur les rythmes scolaires peut être considéré comme une tentative pour desserrer l'étreinte scolaire, pour ouvrir la formation des jeunes vers des finalités culturelles et sociales plus larges.

31 Telles sont les principales questions aujourd'hui en discussion en France dans le domaine de l'éducation et de la scolarisation. Certes, d'autres questions retiennent l'intérêt des autorités, des chercheurs, de tel ou tel secteur de la société : les relations entre la famille et l'école, les différences entre filles et garçons (les filles réussissant mieux à l'école mais étant orientées vers des filières socialement moins rentables), la redéfinition des contenus d'enseignement et des programmes, l'impact des nouvelles technologies, la lutte contre un illettrisme qui n'a pas été éradiqué, le problème d'un apprentissage des langues étrangères rendu plus urgent par la construction de l'Europe, et quelques autres questions, font l'objet de réflexions, de recherches, de décisions mais ne sont pas pour autant au centre des débats publics. Cela ne veut pas dire que ces questions soient moins importantes que celles que nous avons évoquées; elles sont seulement moins en prise avec les contradictions qui traversent la société française et son école. Or, lorsqu'elle débat de l'école, une société débat aussi et avant tout de sa représentation d'elle-même et des contradictions qu'elle doit affronter.

\section{RÉSUMÉS}

En France, toujours autant de passion à propos de l'école. Cependant les objets de débats se déplacent et de nouvelles problématiques apparaissent: comment gérer les progrès de la scolarité et la forte augmentation des bacheliers ? Comment articuler formation et emploi? Comment se protéger de la violence à l'école? Mais, quelles que soient les questions abordées, l'éducation en France reste une affaire d'État.

\section{INDEX}

Mots-clés : politique éducative, système éducatif

Index géographique : France

\section{AUTEUR \\ BERNARD CHARLOT}

Professeur de sciences de l'éducation, université de Paris-VIII, France. 